\title{
Limitation of Use Based on Conditional Approval
}

National Cancer Institute

\section{Source}

National Cancer Institute. Limitation of Use Based on Conditional Approval. NCI

Thesaurus. Code C60654.

A restriction on the use of a device, medicine or treatment based on parameters set forth by the approver. 